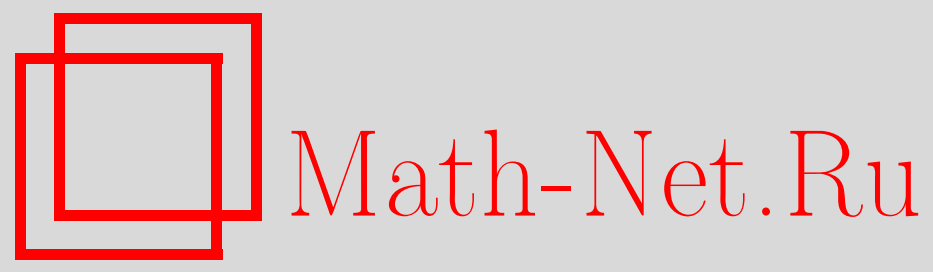

А. Г. Вереникин, Э. Э. Гасанов, Об автоматной детерминизации множеств сверхслов, Дискрет. матем., 2006, том 18, выпуск 2, 84-97

DOI: https://doi.org/10.4213/dm48

Использование Общероссийского математического портала Math-Net.Ru подразумевает, что вы прочитали и согласны с пользовательским соглашением http: //www . mathnet.ru/rus/agreement

Параметры загрузки:

IP : 34.239 .49 .27

26 апреля 2023 г., $11: 58: 19$

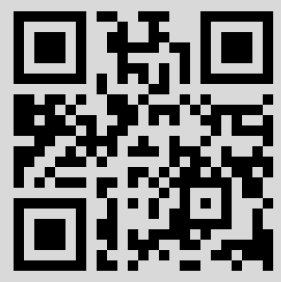


УДК 519.7

\title{
Об автоматной детерминизации множеств сверхслов
}

\author{
() 2006 г. А. Г. Вереникин, Э. Э. Гасанов
}

\begin{abstract}
В работе вводится понятие детерминизируюшего автомата, который для каждого поданного ему на вход сверхслова из заданного множества, начиная с некоторого шага, в каждый момент $t$ выдает значение входного слова в момент $t+1$, то есть предугадывает входное сверхслово. Получен критерий детерминизируемости заданного множества сверхслов - множества, для которого существует детерминизирующий автомат. Приведен наилучший по порядку метод построения детерминизирующего автомата для произвольного детерминизируемого множества сверхслов. Для некоторых детерминизируемых множеств построены оптимальные и асимптотически оптимальные детерминизируюшие автоматы.
\end{abstract}

\section{1. Введение}

Пусть дано некоторое множество сверхслов в алфавите $\{0,1\}$. Автомат детерминизирует это множество, если, для каждого входного слова из этого множества автомат с некоторого момента времени начинает на своем выходе предугадывать значение входного слова в следующий момент. Множество сверхслов детерминизируемо, если существует детерминизирующий его автомат. Показано, что детерминизируемы только множества, состоящие из периодических слов с ограниченным периодом. Под сложностью детерминизирующего автомата понимается число его состояний. Для произвольного детерминизируемого множества получен порядок сложности детерминизирующего его автомата. Для множества сверхслов с фиксированным периодом получено асимптотическое поведение функции сложности детерминизирующего автомата.

Авторы выражают благодарность В. Б. Кудрявцеву за помощь в работе.

\section{2. Основные понятия и формулировка результатов}

Будем использовать следующие обозначения:

$\{0,1\}^{n}-$ множество всех слов длины $n$ в алфавите $\{0,1\}$,

$\{0,1\}^{*}$ - множество всех слов в алфавите $\{0,1\}$, по определению будем считать, что пустое слово $\Lambda$ принадлежит $\{0,1\}^{*}$,

$\{0,1\}^{\infty}-$ множество всех сверхслов в алфавите $\{0,1\}$, 
$|a|$ - длина слова $a \in\{0,1\}^{*}$, по определению $|\Lambda|=0$,

$a(n)-n$-й элемент слова или сверхслова $a$,

$a]_{n}-$ префикс $a$ длины $n$, то есть $\left.a\right]_{n}=a(1) \ldots a(n)$,

${ }_{n}\left[a-\right.$ суффикс слова $a$ длины $n$, то есть ${ }_{n}[a=a(|a|-n+1) \ldots a(|a|)$,

$a b$ - конкатенация слов $a$ и $b$,

$a^{n}=\underbrace{a \ldots a}_{n}-$ конкатенация $n$ слов $a$, здесь $n-$ натуральное число и может быть равно $\infty$,

$\hat{a}-$ слово $a$, записанное в обратном порядке, то есть $\hat{a}=a(|a|) a(|a|-1) \ldots a(1)$,

$m \mid n$ означает, что $m$ - делитель $n$, здесь $m, n$ - натуральные числа.

В статъе рассматриваются конечные автоматы следующего вида:

$$
V=(\{0,1\}, Q,\{0,1\}, \psi, \varphi),
$$

где $\{0,1\}$ - входной алфавит, $Q$ - множество состояний, которое является конечным подмножеством некоторого фиксированного счётного множества, $\{0,1\}$ - выходной алфавит, $\psi:\{0,1\} \times Q \rightarrow Q-$ функщия переходов, $\varphi: 0,1 \times Q \rightarrow\{0,1\}-$ функщия выходов. $V\left(q_{0}\right)-$ инициальный автомат с начальным состоянием $q_{0}$. Таким образом, если на вход автомату $V\left(q_{0}\right)$ подается сверхслово $x$, на выходе получается сверхслово $y$, и $q_{t}$ означает состояние автомата в момент времени $t$, то

$$
\begin{aligned}
y(t) & =\varphi\left(x(t), q_{t-1}\right), \\
q_{t} & =\psi\left(x(t), q_{t-1}\right) .
\end{aligned}
$$

Расширим функции $\psi$ и $\varphi$ на $\{0,1\}^{*} \times Q$, а именно, если на вход автомату $V\left(q_{0}\right)$ подается слово или сверхслово $x$, то $\left.\left.\psi(x]_{t}, q_{0}\right), \varphi(x]_{t}, q_{0}\right)$ означают состояние, в котором находится автомат, и выходное значение в момент времени $t$, соответственно.

Будем говорить, что инициальный автомат $V\left(q_{0}\right)$ детерминизирует множество сверхслов $\mathscr{A} \subset\{0,1\}^{\infty}$, если для любого $a \in \mathscr{A}$ сушествует такое $N$, что для любого $t \geqslant N$ выполняется равенство $\left.\varphi(a]_{t}, q_{0}\right)=a(t+1)$.

Множество сверхслов $\mathscr{A} \subset\{0,1\}^{\infty}$ называется детерминизируемым, если существует детерминизирующий его автомат.

Введем следующие обозначения:

$\omega(V)$ - число состояний автомата $V$,

$\mathbb{G}(\mathscr{A})$ - множество всех конечных инициальных автоматов, детерминизирующих множество сверхслов $\mathscr{A} \subset\{0,1\}^{\infty}$,

$\omega(\mathscr{A})=\min _{G \in \mathbb{G}(\mathscr{A})} \omega(G)$ - минимальное число состояний, достаточное автомату, чтобы детерминизировать $\mathscr{A} \subset\{0,1\}^{\infty}$, если $\mathbb{G}(\mathscr{A})=\varnothing$, то полагаем, что $\omega(\mathscr{A})=\infty$,

$\mathscr{A}^{*}=\{0,1\}^{*} \mathscr{A}=\left\{b a: b \in\{0,1\}^{*}, a \in \mathscr{A}\right\}-$ множество, полученное прибавлением к $\mathscr{A} \subset\{0,1\}^{\infty}$ всевозможных начальных отрезков конечной длины,

$\langle a\rangle-$ множество, состоящее из всех циклических сдвигов слова $a \in\{0,1\}^{*}$, 
$\langle A\rangle=\bigcup_{a \in A}\langle a\rangle$, где $A \subset\{0,1\}^{*}$,

$T(a), T^{\prime}(a) \in\{0,1\}^{*}-$ периодическая и предпериодическая части сверхслова $a \in\{0,1\}^{\infty}$ минимальной длины такие, что $a=T^{\prime}(a)(T(a))^{\infty}$,

$T(\mathscr{A})=\{T(a): a \in \mathscr{A}\}, T^{\prime}(\mathscr{A})=\left\{T^{\prime}(a): a \in \mathscr{A}\right\}$, где $\mathscr{A}-$ некоторое множество периодических сверхслов,

$\tau(a), \tau^{\prime}(a)$ - соответственно, период и предпериод минимальной длины сверхслова $a \in\{0,1\}^{\infty}$, в указанных выше обозначениях $\tau(a)=|T(a)|$ и $\tau^{\prime}(a)=\left|T^{\prime}(a)\right|$.

$\|A\|_{\gamma}=|\{a \in\langle T(\mathscr{A})\rangle: a(1)=\gamma\}|$, где $\mathscr{A}-$ некоторое множество периодических сверхслов, $\gamma \in\{0,1\}$, иными словами $\|\mathscr{A}\|_{\gamma}$ - суммарное число вхождений буквы $\gamma$ в слова из $T(\mathscr{A})$,

$\|\mathscr{A}\|=\max _{\gamma \in\{0,1\}}\|\mathscr{A}\|_{\gamma}$

Определим три параметрических множества сверхслов:

$\mathscr{A}_{\tau \mid n}=\left\{a \in\{0,1\}^{\infty}: \tau(a) \mid n, \tau^{\prime}(a)=0\right\}-$ множество периодических сверхслов с периодом $n$ и нулевым предпериодом,

$\mathscr{A}_{\tau=n}=\left\{a \in\{0,1\}^{\infty}: \tau(a)=n, \tau^{\prime}(a)=0\right\}-$ множество периодических сверхслов с минимальным периодом $n$ и нулевым предпериодом,

$\mathscr{A}_{\tau \leqslant n}=\left\{a \in\{0,1\}^{\infty}: \tau(a) \leqslant n, \tau^{\prime}(a)=0\right\}$ - множество периодических сверхслов с периодом, не большим $n$, и нулевым предпериодом.

Сформулируем основные результаты.

Теорема 1. Множество сверхслов $\mathscr{A} \subset\{0,1\}^{\infty}$ детерминизируемо тогда и только тогда, когда оно состоит из периодчческих сверхслов с ограниченным периодом.

Теорема 2. Если $\mathscr{A}$ детерминизируемо, то

$$
|\langle T(\mathscr{A})\rangle| / 2 \leqslant\|\mathscr{A}\| \leqslant \omega\left(\mathscr{A}^{*}\right) \leqslant|\langle T(\mathscr{A})\rangle| .
$$

Теорема 3. Если $n \geqslant 1$, то $\omega\left(\mathscr{A}_{\tau \mid n}\right)=\omega\left(\mathscr{A}_{\tau \mid n}^{*}\right)=2^{n-1}$, если $n \rightarrow \infty$, то

$$
\begin{gathered}
\omega\left(\mathscr{A}_{\tau=n}\right) \sim \omega\left(\mathscr{A}_{\tau=n}^{*}\right) \sim 2^{n-1}, \\
2^{n} \lesssim \omega\left(\mathscr{A}_{\tau \leqslant n}\right) \lesssim 3 \cdot 2^{n-1} .
\end{gathered}
$$

\section{3. Критерий детерминизируемости и порядок сложности детерминизирующего автомата}

Лемма 1. Если $A$-некоторое множество периодических сверхслов, то

$$
\omega(\mathscr{A}) \geqslant\|\mathscr{A}\| \text {. }
$$


Доказательство. Пусть $f$ - такая частичная детерминированная функщия, определенная на множестве всех начал сверхслов (включая сами сверхслова) из $\mathscr{A}$, что для любого $x \in \mathscr{A}$ найдется такое $N_{x} \geqslant 0$, что выполнено условие

$$
f(x)\left(t+N_{x}\right)=x\left(t+N_{x}+1\right), \quad t \geqslant 0 .
$$

Если существует доопределение $f$ до всюду определенной ограниченно-детерминированной функции, то оно будет задавать инициальный автомат из $\mathbb{G}(\mathscr{A})$. При этом число состояний у такого автомата не меньше числа различимых остаточных функцй функщии $f$ (см. $[1,2])$. Будем обозначать через $f_{a}$ остаточную функщию для $f$, соответствующую слову $a \in\{0,1\}^{*}$, то есть $f_{a}(x) \equiv f(a x)$.

Фиксируем $\gamma \in\{0,1\}$. Возьмем такие $a_{1}, a_{2} \in \mathscr{A}$, что

$$
T\left(a_{1}\right) \neq T\left(a_{2}\right), \quad T\left(a_{1}\right)(1)=T\left(a_{2}\right)(1)=\gamma .
$$

Пусть

$$
b_{1}=\left(T\left(a_{1}\right)\right)^{\tau\left(a_{2}\right)}, \quad b_{2}=\left(T\left(a_{2}\right)\right)^{\tau\left(a_{1}\right)} .
$$

Тогда $\left|b_{1}\right|=\left|b_{2}\right|$ и $b_{1}, b_{2}$ представляются в виде

$$
b_{1}=\gamma b c_{1}, \quad b_{2}=\gamma b c_{2},
$$

где $\left|c_{1}\right|=\left|c_{2}\right|>0, c_{1}(1) \neq c_{2}(1)$. Действительно, если предположить, что $b_{1}=b_{2}$, то получим, что либо $T\left(a_{1}\right)=T\left(a_{2}\right)$, либо $T\left(a_{1}\right)$ и $T\left(a_{2}\right)$ периодические, чего не может быть, так как $T\left(a_{i}\right)$ - минимальная периодическая часть сверхслова $a_{i}, i=1,2$.

Пусть $f_{i}=f_{\left.a_{i}\right]_{i}}$, где

$$
t_{i}=\min \left\{\tau^{\prime}\left(a_{i}\right)+l \tau\left(a_{i}\right) \geqslant N_{a_{i}}: l \in \mathbf{N}\right\}, \quad i=1,2 .
$$

Тогда из (1) и (2) следует, что

$$
f_{1}(\gamma b)=b c_{1}(1) \neq b c_{2}(1)=f_{2}(\gamma b)
$$

то есть, $f_{1}$ и $f_{2}$ отличимы.

Рассмотрим $a \in \mathscr{A}$. Поскольку $T(a)-$ непериодическое слово, справедливо равенство $|\langle T(a)\rangle|=|T(a)|$. Обозначим $k=\|\{a\}\|_{\gamma}$ число вхождений $\gamma$ в слово $T(a)$. Пусть $g_{1}=f_{a]_{i_{1}}}, \ldots, g_{k}=f_{a]_{i_{k}}}$, где

$$
\begin{gathered}
a\left(i_{t}+1\right)=\gamma, \quad 1 \leqslant t \leqslant k, \quad t_{3} \leqslant i_{1}<\ldots<i_{k} \leqslant t_{3}+\tau(a)-1, \\
t_{3}=\min \left\{\tau^{\prime}(a)+l \tau(a) \geqslant N_{a}: l \in \mathbf{N}\right\} .
\end{gathered}
$$

Как и выше, легко показывается, что $g_{1}, \ldots, g_{k}$ попарно различимы.

Таким образом, число попарно различимых остаточных функций функции $f$ не меньше, чем $\|\mathscr{A}\|_{\gamma}$. Взяв максимум по $\gamma$, получаем утверждение леммы.

Лемма 2. Если $\mathscr{A}$ - некоторое множество периодических сверхслов, то

$$
\omega(\mathscr{A}) \geqslant \frac{|\langle T(\mathscr{A})\rangle|}{2} .
$$


Доказательство. Легко видеть, что

$$
\|\mathscr{A}\|=\max _{\gamma \in\{0,1\}}\|\mathscr{A}\|_{\gamma} \geqslant \frac{|\langle T(\mathscr{A})\rangle|}{2} .
$$

Отсюда, учитывая лемму 1, получаем утверждение леммы 2.

Но здесь мы приведем другое доказательство леммы 2, которое проясняет одно важное свойство детерминизирующего автомата.

Заметим, что для любых двух сверхслов $a, b \in \mathscr{A}$ либо $\langle T(a)\rangle=\langle T(b)\rangle$, то есть $T(a)$ и $T(b)$ получаются друг из друга циклическим сдвигом, либо $\langle T(a)\rangle \cap\langle T(b)\rangle=\varnothing$.

Если множество $\mathscr{A}$ не детерминизируемо, то $\omega(\mathscr{A})=\infty$ и утверждение леммы справедливо.

Предположим, что множество $\mathscr{A}$ детерминизируемо и

$$
G\left(q_{0}\right)=\left(\{0,1\}, Q,\{0,1\}, \psi, \varphi, q_{0}\right)
$$

- детерминизирующий его автомат. Пусть этот автомат задан диаграммой Мура. Ребро $e$ диаграммы Мура можно задавать парой $(q, \gamma)$, где $q$ - вершина диаграммы Мура, из которой исходит ребро, $\gamma$ - входная буква, приписанная ребру (в таком случае будем писать $e=(q, \gamma))$. Через $\varphi(e)$ и $\psi(e)$ будем обозначать соответственно выходную букву, соответствующую ребру $e$, и вершину диаграммы Мура, в которую ведет ребро $e$.

Пусть $a_{1}, a_{2} \in \mathscr{A}$ - такие сверхслова, что $\left\langle T\left(a_{1}\right)\right\rangle \cap\left\langle T\left(a_{2}\right)\right\rangle=\varnothing$.

Фиксируем $i \in\{1,2\}$. Пусть $e_{i}$ - последовательность ребер диаграммы Мура, соответствующая входному сверхслову $a_{i}$, то есть для любого $t \geqslant 1$ выполнено равенство $\left.e_{i}(t)=\left(\psi\left(a_{i}\right]_{t-1}, q_{0}\right), a_{i}(t)\right)$. Здесь $\left.\psi\left(a_{i}\right]_{0}, q_{0}\right)=q_{0}$. Так как $a_{i}$ - периодическое слово и $Q$ - конечное множество, последовательность $e_{i}$ периодическая. Пусть $l_{i}-$ период последовательности $e_{i}$ и $e_{i}^{\prime}(1) e_{i}^{\prime}(2) \ldots e_{i}^{\prime}\left(l_{i}\right)$ - периодическая ее часть. Пусть $e_{i}^{\prime}(t)=\left(q_{i}(t), \gamma_{i}(t)\right), t=1,2, \ldots, l_{i}$.

Слово $\gamma_{i}(1) \gamma_{i}(2) \ldots \gamma_{i}\left(l_{i}\right)$ периодическое с периодом $\tau\left(a_{i}\right)$, поэтому

$$
l_{i} \geqslant \tau_{a_{i}}=\left|\left\langle T\left(a_{i}\right)\right\rangle\right| .
$$

По определению $\psi\left(e_{i}^{\prime}(t)\right)=q_{i}(t+1)$ для каждого $t \in\left\{1,2, \ldots, l_{i}\right\}$ (здесь и далее считаем, что $\left.q_{i}\left(l_{i}+1\right)=q_{i}(1), \gamma_{i}\left(l_{i}+1\right)=\gamma_{i}(1), e_{i}\left(l_{i}+1\right)=e_{i}(1)\right)$. Так как $G\left(q_{0}\right)-$ детерминизирующий автомат, то $\varphi\left(e_{i}^{\prime}(t)\right)=\gamma_{i}(t+1)$ для каждого $t \in\left\{1,2, \ldots, l_{i}\right\}$.

Покажем, что множества ребер $\left\{e_{1}^{\prime}(1), e_{1}^{\prime}(2), \ldots, e_{1}^{\prime}\left(l_{1}\right)\right\}$ и $\left\{e_{2}^{\prime}(1), e_{2}^{\prime}(2), \ldots, e_{2}^{\prime}\left(l_{2}\right)\right\}$ не пересекаются. Предположим обратное, то есть пусть существуют $t_{i} \in\left\{1,2, \ldots, l_{i}\right\}$, $i=1,2$, такие, что $e_{1}^{\prime}\left(t_{1}\right)=e_{2}^{\prime}\left(t_{2}\right)$. Так как $\left\langle T\left(a_{1}\right)\right\rangle \cap\left\langle T\left(a_{2}\right)\right\rangle=\varnothing$, без ограничения общности можно считать, что $e_{1}^{\prime}\left(t_{1}+1\right) \neq e_{2}^{\prime}\left(t_{2}+1\right)$. Понятно, что $q_{1}\left(t_{1}+1\right)=q_{2}\left(t_{2}+1\right)$, следовательно, $\gamma_{1}\left(t_{1}+1\right) \neq \gamma_{2}\left(t_{2}+1\right)$, и значит, $\varphi\left(e_{1}^{\prime}\left(t_{1}\right)\right) \neq \varphi\left(e_{2}^{\prime}\left(t_{2}\right)\right)$, откуда следует, что $e_{1}^{\prime}\left(t_{1}\right) \neq e_{2}^{\prime}\left(t_{2}\right)$. Получаем противоречие.

Следовательно, так как $a_{1}, a_{2} \in \mathscr{A}$ - произвольные сверхслова такие, что $\left\langle T\left(a_{1}\right)\right\rangle \cap\left\langle T\left(a_{2}\right)\right\rangle=\varnothing$, число ребер в диаграмме Мура автомата $G\left(q_{0}\right)$ не меньше, чем $|\langle T(\mathscr{A})\rangle|$. А так как из каждой вершины диаграммы Мура исходит ровно два ребра, число состояний автомата $G\left(q_{0}\right)$ не меньше, чем $|\langle T(\mathscr{A})\rangle| / 2$.

Лемма доказана.

Лемма 3. Пусть $G=(\{0,1\}, Q,\{0,1\}, \psi, \varphi)-$ автомат. Тогда, если $G(q) \in \mathbb{G}(\mathscr{A})$ для любого $q \in Q$, то $G(q) \in \mathbb{G}\left(\mathbb{A}^{*}\right)$ для любого $q \in Q$. 
Доказательство. Пусть $G(q) \in \mathbb{G}((\mathscr{A}))$ для любого $q \in Q$. Возьмем произвольное состояние $q^{\prime} \in Q$ и произвольное слово $a \in \mathscr{A}^{*}$. По определению $a=b c$, где $b \in\{0,1\}^{*}$, $c \in \mathscr{A}$. Пусть $\psi\left(b, q^{\prime}\right)=q^{\prime \prime}$. Так как автомат $G\left(q^{\prime \prime}\right)$ детерминизирует слово $c \in \mathscr{A}$, автомат $G\left(q^{\prime}\right)$ детерминизирует слово $a=b c \in \mathbb{A}^{*}$. В силу произвольности слова $a$ справедливо включение $G\left(q^{\prime}\right) \in \mathbb{G}\left(\mathscr{A}^{*}\right)$.

Лемма доказана.

Лемма 4. Если $\mathscr{A}$ - некоторое множество периодических сверхслов, то

$$
\omega\left(\mathscr{A}^{*}\right) \leqslant|\langle T(\mathscr{A})\rangle| \text {. }
$$

Доказательство. Пусть

$$
\mathscr{B}=\left\{(T(a))^{\infty}: a \in \mathscr{A}\right\}
$$

то есть $\mathscr{B}$ получено из $\mathscr{A}$ отбрасыванием предпериодической части у сверхслов из $\mathscr{A}$. Понятно, что $\mathscr{A}^{*}=\mathscr{B}^{*}$ и $|\langle T(\mathscr{A})\rangle|=|\langle T(\mathscr{B})\rangle|$, поэтому сразу можно считать, что $T^{\prime}(\mathscr{A})=\{\Lambda\}$.

Пусть $\alpha \in\{0,1\}, u, q \in\{0,1\}^{*}$. Введем обозначение

$$
\left.\left.r(\alpha, u, q)=\max \left\{l:\left(\alpha u^{\infty}\right)\right]_{l}=\left(q^{\infty}\right)\right]_{l}, l \in\{0,1,2, \ldots\}\right\}
$$

Здесь $r(\alpha, u, q) \leqslant 1$, если $u=\Lambda, r(\alpha, u, q)=0$, если $q=\Lambda$, и $r(\alpha, u, q)=\infty$, если $\alpha u^{\infty}=q^{\infty}, u \neq \Lambda, q \neq \Lambda$. Функция $r(\alpha, u, q)$ говорит, на сколько совпадают начала слов $\alpha u^{\infty}$ и $q^{\infty}$.

Введем обозначения

$$
\begin{aligned}
Q(\mathscr{A}) & =\{\hat{q}: q \in\langle T(\mathscr{A})\rangle\}, \\
R(\alpha, u) & =\max _{q \in Q(\mathscr{A})} r(\alpha, u, q), \\
A(\alpha, u) & =\{q \in Q(\mathscr{A}): r(\alpha, u, q)=R(\alpha, u)\} .
\end{aligned}
$$

Через $s(q)=(q(|q|) q)]_{|q|}$ обозначим циклический сдвиг слова $q$ на единицу вправо.

Легко видеть, что для всех $q \in Q(\mathscr{A})$ выполняется соотношение

$$
A(q(|q|), q)=\{s(q)\}
$$

Поскольку мы предположили, что $T^{\prime}(\mathscr{A})=\{\Lambda\}$, для любого $x \in \mathscr{A}$ существует такое слово $q^{\prime} \in Q(\mathscr{A})$, что $q^{\prime}=\widehat{T}(x)$, то есть $x=\left(\widehat{q^{\prime}}\right)^{\infty}$. Отсюда следует, что для любого $t \geqslant 1$ существует такое слово $q^{\prime \prime} \in Q(\mathscr{A})$, что

$$
\left.\left(q^{\prime \prime}\right)^{\infty}\right]_{t}=\widehat{x]_{t}}
$$

причем в качестве $q^{\prime \prime}$ можно взять слово, полученное из $q^{\prime}$ циклическим сдвигом вправо на остаток от деления $t$ на $\tau(x)=\left|q^{\prime}\right|$.

Рассмотрим отображение $\Psi:\{0,1\} \times\{0,1\}^{*} \rightarrow Q(\mathscr{A})$ такое, что $\Psi(\alpha, u) \in A(\alpha, u)$, $\alpha \in\{0,1\}, u \in\{0,1\}^{*}$.

Рассмотрим автомат

$$
G_{\mathscr{A}}=(\{0,1\}, Q(\mathscr{A}),\{0,1\}, \psi, \varphi)
$$


с каноническими уравнениями

$$
\begin{aligned}
q_{t} & =\psi\left(x(t), q_{t-1}\right)=\Psi\left(x(t), q_{t-1}\right), \\
y(t) & =\varphi\left(x(t), q_{t-1}\right)=q_{t}\left(\left|q_{t}\right|\right) .
\end{aligned}
$$

Отметим, что если $q_{t-1}\left(\left|q_{t-1}\right|\right)=x(t)$, то согласно (3) $q_{t}=s\left(q_{t-1}\right)$.

Покажем по индукции, что для любых $t \geqslant 1, x \in \mathscr{A}$ и $q_{0} \in Q(\mathscr{A})$ выполнено равенство

$$
\left.\left(q_{t}\right)^{\infty}\right]_{t}=\widehat{x]_{t}}
$$

это означает, что каждое текущее состояние помнит все поступившие буквы входного слова.

В качестве базы индукции рассмотрим случай $t=1$. В этом случае

$$
q_{1}=\Psi\left(x(1), q_{0}\right) \in A\left(x(1), q_{0}\right) .
$$

Ясно, что в $Q(\mathcal{A})$ есть состояние, начинающееся с $x(1)$, например, $s(\widehat{T}(x))$, поэтому $q_{1}(1)=x(1)$.

Проведем индуктивный переход. По предположению индукции

$$
\left.\left(q_{t-1}\right)^{\infty}\right]_{t-1}=\widehat{x]_{t-1}}
$$

Следовательно,

$$
\left.x(t)\left(\left(q_{t-1}\right)^{\infty}\right]_{t-1}\right)=x(t) \widehat{x]_{t-1}}=\widehat{x]_{t}} .
$$

Поскольку согласно (4) существует $q^{\prime} \in Q(\mathscr{A})$ такое, что $\left.\left(q^{\prime}\right)^{\infty}\right]_{t}=\widehat{x]_{t}}$, для $q_{t}=\Psi\left(x(t), q_{t-1}\right) \in A\left(x(t), q_{t-1}\right)$ справедливо равенство $\left.\left(q_{t}\right)^{\infty}\right]_{t}=\widehat{x]_{t}}$.

Тем самым равенство (6) доказано.

Введем обозначение $q^{\prime}=\hat{T}(x)$. Из равенства (6) следует, что при достаточно больших $t$ (например, при $t>\left|q^{\prime}\right|+\max _{q \in Q(s)}|q|$ ) состояние $q_{t}$ определяется однозначно и это состояние, полученное из $q^{\prime}$ циклическим сдвигом вправо на остаток от деления $t$ на $\left|q^{\prime}\right|$, то есть $q_{t}=x(t) x(t-1) \ldots x(t-\tau(x)+1)$. Поэтому

$$
y(t)=q_{t}\left(\left|q_{t}\right|\right)=x(t-\tau(x)+1)=x(t+1) .
$$

Таким образом, мы показали, что для любого $q_{0}$ автомат $G_{\mathscr{A}}\left(q_{0}\right) \in \mathbb{G}(\mathscr{A})$, откуда согласно лемме 3 справедливо включение $G_{\mathscr{A}}\left(q_{0}\right) \in \mathbb{G}\left(\mathscr{A}^{*}\right)$.

В доказательстве леммы 4 содержится оптимальный по порядку метод построения детерминизирующего автомата для произвольного детерминизируемого множества периодических сверхслов. Суть алгоритма в следующем. В качестве алфавита состояний выбираем $Q(\mathscr{A})$ - записанные в обратном порядке все циклические сдвиги периодических частей сверхслов из $\mathscr{A}$. Из состояния $q$ по $x(t)$ переходим в такое состояние $q^{\prime}$, что слова $x(t) q^{\infty}$ и $\left(q^{\prime}\right)^{\infty}$ имеют общий префикс наибольшей длины. Множество всех таких $q^{\prime}$ обозначаем $A(x(t), q)$. Если $x(t)=q(|q|)$, то $q^{\prime}$ представляет собой циклический сдвиг вправо слова $q$. Выходное значение $y(t)$ полагаем равным $q^{\prime}\left(\left|q^{\prime}\right|\right)$.

Пример 1. Пусть $\mathscr{A}=\left\{0^{\infty},(01)^{\infty},(001)^{\infty}\right\}$. Тогда $Q(\mathscr{A})=\{0,01,10,001,010,100\}$. Значения для $A(\overline{q(|q|)}, q)$ определены в табл. 1. На рис. 1 приведена диаграмма Мура автомата, построенного по указанному методу. Пунктиром обозначены переходы из $q$ по $\overline{q(|q|)}$, a сплошными линиями - по $q(|q|)$. Полученный после минимизации автомат изображен на рис. 2. Число его состояний равно четырем, что совпадает с $\|\mathscr{A}\|=\max \left\{\|\mathscr{A}\|_{0},\|\mathscr{A}\|_{1}\right\}=$ $\max \{2,4\}=4$. Таким образом, построенный автомат детерминизирует $\mathscr{A}^{*}$ и является оптимальным по числу состояний. 
Таблица 1.

\begin{tabular}{|c|c|c|c|c|c|c|}
\hline$q$ & 0 & 01 & 10 & 001 & 010 & 100 \\
\hline$A(q(|q|), q)$ & $\{100\}$ & $\{001\}$ & $\{10,100\}$ & $\{0\}$ & $\{10\}$ & $\{10,100\}$ \\
\hline
\end{tabular}

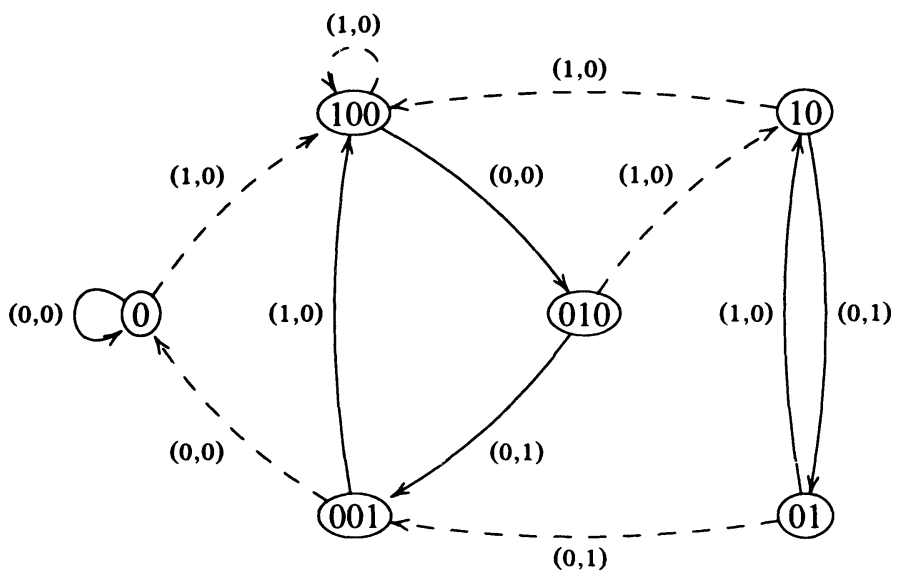

Рис. 1. До минимизации

Доказательство теоремы 1. Докажем необходимость. Пусть $\mathscr{A}$ детерминизируемо и $G\left(q_{0}\right)=(\{0,1\}, Q,\{0,1\}, \psi, \varphi)-$ детерминизирующий его автомат. Тогда для любого $x \in \mathscr{A}$ найдется такое $N$, что $\left.x(t+1)=\varphi(x]_{t}, q_{0}\right), t \geqslant N$, и сверхслово $x(N+1) x(N+2) \ldots$ можно рассматривать, как генерируемое конечным автономным автоматом. Оно периодично $([1,2])$. Следовательно, $x$ также периодично. Ограниченность периода вытекает непосредственно из леммы 1.

Докажем достаточность. Пусть $\mathscr{A}$ состоит из периодических сверхслов с ограниченным периодом. Тогда по лемме 4 существует детерминизирующий его автомат с количеством состояний, не превосходящим $|\langle T(\mathscr{A})\rangle|<\infty$.

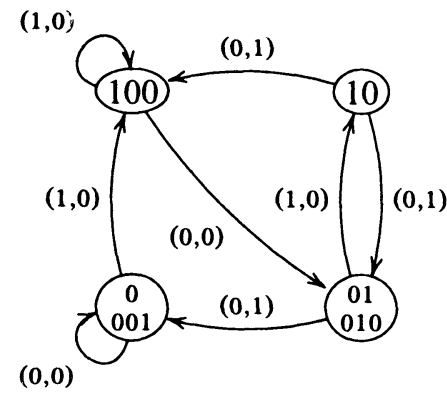

Рис. 2. После минимизации 
Объединяя леммы 1 и 4 с доказанной теоремой и учитывая, что $|\langle T(\mathscr{A})\rangle| / 2 \leqslant\|\mathscr{A}\|$, получаем теорему 2.

\section{4. Оценки сложности детерминизирующего автомата для некоторых множеств}

Функщия $\mu: \mathbf{N} \rightarrow\{-1,0,1\}$, определяемая равенством

$$
\mu(n)= \begin{cases}1, & \text { если } n=1, \\ 0, & \text { если } p^{2} \mid n, \text { где } p \text { - простое число, } \\ (-1)^{k}, & \text { если } n=p_{1} \ldots p_{k}, \text { где } p_{1}, \ldots, p_{k}-\text { различные простые числа }\end{cases}
$$

называется функцией Мёбиуса.

Следующая теорема дает формулу обращения Мёбиуса $[3,4]$.

Теорема 4. Если для функций $f: \mathbf{N} \rightarrow \mathbf{Z}, g: \mathbf{N} \rightarrow \mathbf{Z}$ справедливо равенство

$$
f(n)=\sum_{d \mid n} g(d),
$$

mo

$$
g(n)=\sum_{d \mid n} \mu(d) f(n / d) .
$$

Обозначим через $\mathscr{R}_{n}$ множество всех непериодических слов из $\{0,1\}^{n}$.

Лемма 5. Справедливы следуючие соотношения.

(1) $\left|\mathscr{R}_{n}\right|=\sum_{d \mid n} \mu(d) 2^{n / d}$, где $\mu-$ функция Мёбиуса.

(2) $\left|\mathscr{R}_{n}\right| \sim 2^{n}$ npu $n \rightarrow \infty$.

(3) $\sum_{k=1}^{n}\left|\mathscr{R}_{k}\right| \sim 2^{n+1}$ npu $n \rightarrow \infty$.

Доказательство. Докажем первое утверждение. Ясно, что

$$
\{0,1\}^{n}=\bigsqcup_{d \mid n}\left\{a^{n / d}: a \in \mathscr{R}_{d}\right\},
$$

откуда

$$
2^{n}=\sum_{d \mid n}\left|\mathscr{R}_{d}\right|
$$

Применив формулу обращения Мёбиуса, получаем искомое равенство. 
Докажем второе утверждение. Из (7) получаем, что

$$
\begin{aligned}
2^{n} & \geqslant\left|\mathscr{R}_{n}\right|=2^{n}-\sum_{d \mid n, d<n}\left|\Re_{d}\right|=2^{n}-\sum_{d \mid n, d \leqslant[n / 2]}\left|\mathscr{R}_{d}\right| \\
& \geqslant 2^{n}-\sum_{d \leqslant[n / 2]}\left|\mathscr{R}_{d}\right| \\
& \geqslant 2^{n}-2^{[n / 2]+1} .
\end{aligned}
$$

Отсюда следует, что $\left|\mathscr{R}_{n}\right| \sim 2^{n}$ при $n \rightarrow \infty$.

Третье утверждение следует из второго.

Лемма 6. Справедливы оченки

(1) $\omega\left(\mathscr{A}_{\tau \mid n}\right) \geqslant 2^{n-1}$

(2) $\omega\left(\mathscr{A}_{\tau=n}\right) \geqslant \frac{1}{2}\left|\mathscr{R}_{n}\right|$,

(3) $\omega\left(\mathscr{A}_{\tau \leqslant n}\right) \geqslant \frac{1}{2} \sum_{k=1}^{n}\left|\mathscr{R}_{k}\right|$.

Доказательство. Согласно лемме 2

$$
\begin{aligned}
& \omega\left(\mathscr{A}_{\tau \mid n}\right) \geqslant \frac{1}{2}\left|\left\langle T\left(\mathscr{A}_{\tau \mid n}\right)\right\rangle\right|=\frac{1}{2}\left|T\left(\mathscr{A}_{\tau \mid n}\right)\right|=\frac{1}{2}\left|\{0,1\}^{n}\right|=2^{n-1}, \\
& \omega\left(\mathscr{A}_{\tau=n}\right) \geqslant \frac{1}{2}\left|\left\langle\mathscr{A}_{\tau=n}\right\rangle\right|=\frac{1}{2}\left|T\left(\mathscr{A}_{\tau=n}\right)\right|=\frac{1}{2}\left|\mathscr{R}_{n}\right|, \\
& \omega\left(\mathscr{A}_{\tau \leqslant n}\right) \geqslant \frac{1}{2}\left|\left\langle\mathscr{A}_{\tau \leqslant n}\right\rangle\right|=\frac{1}{2}\left|T\left(\mathscr{A}_{\tau \leqslant n}\right)\right|=\frac{1}{2}\left|\bigsqcup_{k=1}^{n}\right| \mathscr{R}_{k}\left|=\frac{1}{2} \sum_{k=1}^{n}\right| \mathscr{R}_{k} \mid,
\end{aligned}
$$

что доказывает все три оценки.

Зададим автомат $\xi^{n}$ последовательностью из $n$ задержек. Будем считать, что $\mathfrak{F}^{0}$ задает тождественное отображение. Автомат $\xi^{n}=\left(\{0,1\},\{0,1\}^{n},\{0,1\}, \psi, \varphi\right)$ задается следующими каноническими уравнениями:

$$
\begin{aligned}
\varphi\left(x(t), q_{t-1}\right) & =q_{t-1}(1), \\
\psi\left(x(t), q_{t-1}\right) & =q_{t-1}(2) \ldots q_{t-1}(n) x(t) .
\end{aligned}
$$

Очевидно, что

$$
\omega\left(G^{n}\right)=2^{n}
$$

На рис. 3 и 4 приведены диаграммы Мура для $\oiint^{1}$ и $\oiint^{2}$.

Лемма 7. Справедливы соотношения

$$
\begin{aligned}
\omega\left(\mathscr{A}_{\tau \mid n}\right) & \leqslant \omega\left(\mathscr{A}_{\tau \mid n}^{*}\right) \leqslant 2^{n-1}, \\
\omega\left(\mathscr{A}_{\tau=n}\right) & \leqslant \omega\left(\mathscr{A}_{\tau=n}^{*}\right) \leqslant 2^{n-1} .
\end{aligned}
$$


$(1,0)$

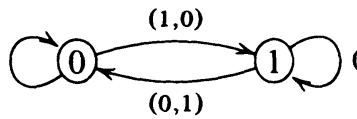

Рис. 3. $\mathscr{G}^{1}$ детерминизирует $\mathbb{A}_{\tau \mid 2}$

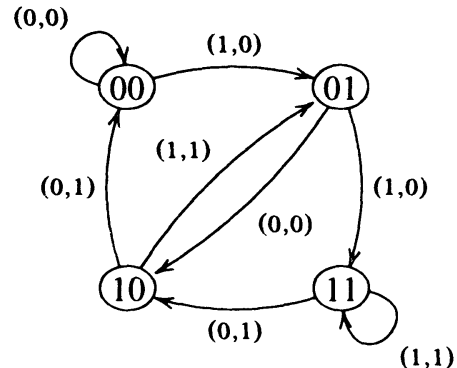

Рис. 4. $\mathscr{G}^{2}$ детерминизирует $\mathscr{A}_{\tau \mid 3}$

Доказательство. Пусть

$$
G^{n-1}\left(q_{0}\right)=\left(\{0,1\}, Q,\{0,1\}, \psi, \varphi, q_{0}\right) .
$$

Для любого $x \in \mathscr{A}_{\tau \mid n}^{*}$

$$
\left.\varphi(x]_{t}, q_{0}\right)=x(t-(n-1))=x(t-n+1)=x(t+1), \quad t \geqslant n+\tau^{\prime}(x) .
$$

Отсюда $\mathbb{F}^{n-1}\left(q_{0}\right) \in \mathbb{G}\left(\mathscr{A}_{\tau \mid n}^{*}\right)$. Остается заметить, что $\mathbb{G}\left(\mathscr{A}_{\tau=n}^{*}\right) \subset \mathbb{G}\left(\mathscr{A}_{\tau \mid n}^{*}\right)$.

Автомат $\mathscr{G}^{n-1}$ не является оптимальным для множества $\mathscr{A}_{\tau=n} ;$ так, на рис. 5,6 и 7,8 приведены примеры оптимальных по числу состояний автоматов, детерминизирующих $\mathscr{A}_{\tau=3}$ и $\mathscr{A}_{\tau=4}$ соответственно.

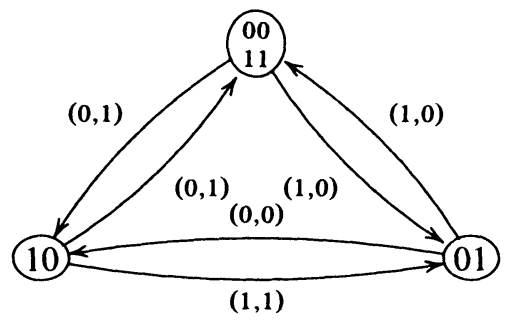

Рис. 5. Пример автомата, детерминизирующего $\mathscr{A}_{\tau=3}$ 


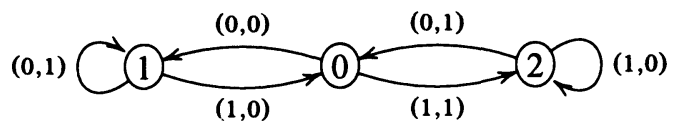

Рис. 6. Пример автомата, детерминизирующего $\mathscr{A}_{\tau=3}$

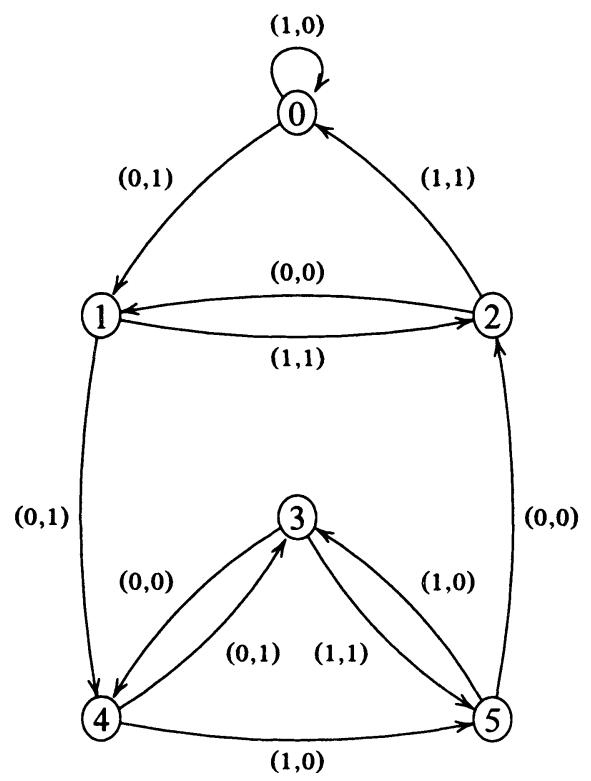

Рис. 7. Пример автомата, детерминизирующего $\mathscr{A}_{\tau=4}$

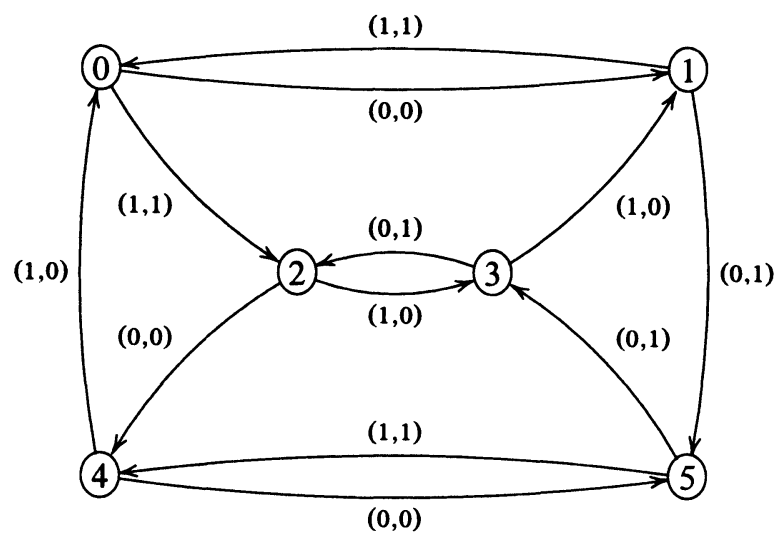

Рис. 8. Пример автомата, детерминизирующего $A_{\tau=4}$ 
Лемма 8. Справедливо неравенство

$$
\omega\left(\mathscr{A}_{\tau \leqslant n}\right) \leqslant 3 \cdot 2^{n-1} .
$$

Доказательство. Рассмотрим инициальный автомат

$$
\Sigma_{n}\left(*^{n}\right)=\left(\{0,1\}, Q,\{0,1\}, \psi, \varphi, *^{n}\right), \quad Q \subset\{0,1, *, \#\}^{n},
$$

определенный каноническими уравнениями

$$
\begin{aligned}
& y(t)= \begin{cases}0, & \text { если } q(t-1)=*^{n}, \\
\alpha_{1}, & \text { если } q(t-1)=*^{n-1} \alpha_{1}, \\
\alpha_{2}, & \text { если } q(t-1)=*^{n-k} \alpha_{1} \alpha_{2} \ldots \alpha_{k}, 2 \leqslant k \leqslant n-1, \\
\alpha_{1}, & \text { если } q(t-1)=\# \alpha_{1} \ldots \alpha_{n-1},\end{cases} \\
& q(t)= \begin{cases}*^{n-1} x(t), & \text { если } q(t-1)=*^{n}, \\
*^{n-k} \alpha_{2} \ldots \alpha_{k} x(t), & \text { если } q(t-1)=*^{n-k} \alpha_{1} \ldots \alpha_{k}, x(t)=\alpha_{1}, n-k \geqslant 1, \\
*^{n-k-1} \alpha_{1} \ldots \alpha_{k} x(t), & \text { если } q(t-1)=*^{n-k} \alpha_{1} \ldots \alpha_{k}, x(t) \neq \alpha_{1}, n-k \geqslant 2, \\
\# \alpha_{2} \ldots \alpha_{n-1} x(t), & \text { если } q(t-1)=* \alpha_{1} \ldots \alpha_{n-1}, x(t) \neq \alpha_{1}, \\
\# \alpha_{2} \ldots \alpha_{n-1} x(t), & \text { если } q(t-1)=\# \alpha_{1} \ldots \alpha_{n-1},\end{cases} \\
& q(0)=*^{n} .
\end{aligned}
$$

Если $x \in \mathscr{A}_{\tau \leqslant n}$ и $\tau(x)=\tau$, то для любого $k, 1 \leqslant k \leqslant \tau-1$, найдется такое $t$, что $x(t-k) \neq x(t)$. С другой стороны, $x(t-\tau)=x(t)$ для всех $t \geqslant \tau+1$. Поэтому при достаточно больших $t$

$$
q(t-1)= \begin{cases}*^{n-\tau} x(t-\tau) \ldots x(t-1), & \text { если } \tau \leqslant n-1, \\ \# x(t-n+1) \ldots x(t-1), & \text { если } \tau=n,\end{cases}
$$

и следовательно,

$$
y(t)=x(t-\tau(x)+1)=x(t+1)
$$

то есть, $\Sigma_{n} \in \mathbb{G}\left(\mathscr{A}_{\tau \leqslant n}\right)$.

Далее, из включения

$$
Q \subset\left(\bigsqcup_{k=0}^{n-1}\left\{*^{n-k} a: a \in\{0,1\}^{k}\right\}\right) \sqcup\left\{\# a: a \in\{0,1\}^{n-1}\right\},
$$

получаем оценку

$$
\omega\left(\mathscr{A}_{\tau \leqslant n}\right) \leqslant|Q| \leqslant \sum_{k=0}^{n-1} 2^{k}+2^{n-1}=3 \cdot 2^{n-1}-1,
$$

что и требовалось доказать.

Утверждение теоремы 3 есть следствие лемм 6, 7 и 8.

Замечание 1. Построенный в доказательстве леммы 8 автомат $\Sigma_{n}$, детерминизирующий $\mathscr{A}_{\tau \leqslant n}$, не является оптимальным по числу состояний. Например, $\mathcal{G}^{1}$ детерминизирует $\mathscr{A}_{\tau \leqslant 2}$ и имеет 2 состояния, а $\Sigma_{2}$ имеет 5 состояний. На рис. 9 приведена диаграмма Мура автомата, детерминизирующего $\mathscr{A}_{\tau \leqslant 3}$ и имеющего пять состояний (то есть являющегося оптимальным, поскольку $\left\|\mathcal{A}_{\tau \leqslant 3}\right\|=5$ ), в то время как $\Sigma_{3}$ имеет 9 состояний. 


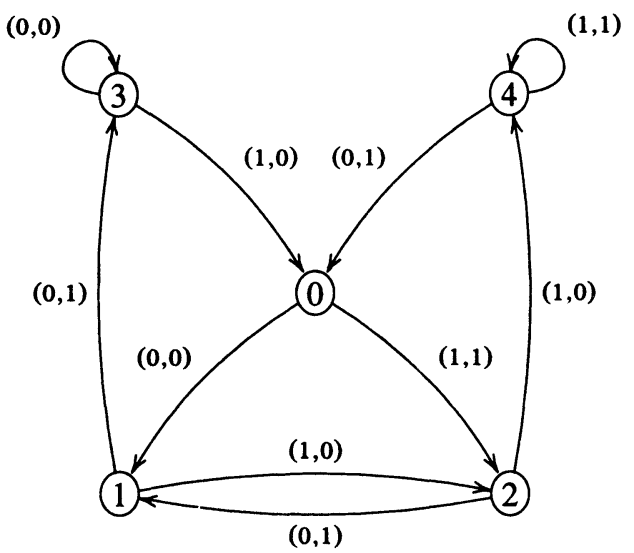

Рис. 9. Пример автомата, детерминизирующего $\mathscr{A}_{\tau \leqslant 3}$

\section{Список литературы}

1. Кудрявцев В. Б., Алешин С. В., Подколзин А. С., Введение в теорию автоматов. Наука, Москва, 1985.

2. Трахтенброт Б. А., Барздинь Я. М., Конечные автоматы (поведение и синтез). Наука, Москва, 1970.

3. Виноградов И. М. Введение в теорию чисел. Наука, Москва, 1981.

4. Кострикин А.И. Основные структуры алгебры. Физматлит, Москва, 2000.

Статья поступила 22.09.2005. 